\title{
Effect of caregivers' expressed emotion on the care burden and rehospitalization rate of schizophrenia
}

This article was published in the following Dove Press journal:

Patient Preference and Adherence

6 September 2017

Number of times this article has been viewed

\section{Xia Wang' \\ Qiongni Chen ${ }^{2}$ \\ Min Yang'}

'Xiangya School of Nursing, ${ }^{2}$ Nursing Department, Second Xiangya Hospital, Central South University, Changsha, Hunan, People's Republic of China

Video abstract

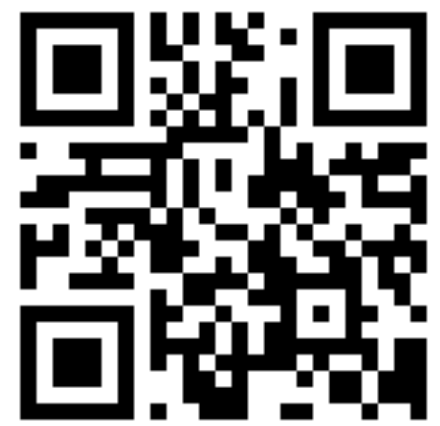

Point your SmartPhone at the code above. If you have a QR code reader the video abstract will appear. Or use: http://youtu.be/2 GQFffzEpU

Correspondence: Min Yang Xiangya School of Nursing, Central South University, 172 Tongzipo

Road, Changsha, Hunan 4I00I3,

People's Republic of China

Tel +86 73I 82650275

Email yangminzhu@csu.edu.cn
Background: This study assessed the effect of expressed emotion (EE) among caregivers of schizophrenia patients on their care burden and the illness rehospitalization rate.

Subjects and methods: A total of 64 schizophrenia patients hospitalized for the first time and their key caregivers were recruited. The Chinese version of the Camberwell Family Interview (CFI-CV) was used to evaluate the EE of the key caregivers. A family burden questionnaire was used to evaluate the care burden. The patients' rehospitalization rate and medication compliance were evaluated by the self-designated criteria. The data collection was carried out at the first meeting in the hospital, at 6 months and 12 months after hospital discharge by using the same instruments.

Results: The subjective stress burden and subjective demand burden scores were higher in caregivers before and after discharge with statistical difference between the various observation time points $(P<0.05)$. Significant differences were observed in the rehospitalization rate between patients with high medication adherence and low medication adherence at 12 months $(P<0.01)$ and between patients with high expressed emotion (HEE) and low expressed emotion (LEE; $P<0.05$ ). The rehospitalization rate in patients with HEE caregivers was higher than that in those with LEE caregivers. The subjective stress burden scores were statistically significant between HEE and LEE caregivers $(P<0.05)$.

Conclusion: HEE is a predictor of rehospitalization rate in schizophrenic patients. The burdens of care scores are high in caregivers of schizophrenic patients. The caregivers with HEE have a high score in burden of care compared with those with LEE.

Keywords: expressed emotion, care burden, schizophrenia, rehospitalization rate, caregivers

\section{Introduction}

Mental illnesses can represent disruptive and disabling conditions for patients and, in the meantime, a severe burden for their family and society. Although antipsychotic drugs are widely used in clinical practice, mental illnesses still have high recurrence rates, which can require frequent hospitalizations. Familial relational conflicts can represent an important factor associated with revolving door hospitalizations. ${ }^{1}$ Family members can positively support patients by providing care directly but, sometimes, can also negatively influence clinical conditions by showing negative emotions toward patients. The construct of expressed emotion (EE), developed in the 1960s and 1970s in England by Brown et $\mathrm{al}^{2}$ and Brown and Rutter, ${ }^{3}$ is an important measure of the patient-relative (or caregiver) relationship. It is represented by a series of specific emotions, attitudes, and behaviors expressed by caregivers toward the patient. ${ }^{4}$

The gold-standard measure of EE is a semi-structured interview known as the Camberwell Family Interview $(\mathrm{CFI})^{5}$ that is conducted with the relatives or caregivers 
of patients. The most important scales assessed by CFI are criticism, hostility, and emotional overinvolvement. The classification of family members as high or low in EE is made on the basis of these scales' rating. Having high levels of EE in the family environment is widely recognized as a robust environmental risk factor for relapse and exacerbation of the illness ${ }^{6-8}$ across numerous countries and cultures. ${ }^{9,10}$ Phillips and Xiong used the CFI scale in China and found that $42.1 \%$ of the family members of schizophrenia patients had high expressed emotion (HEE), but this was not associated with the rehospitalization rate of schizophrenia. ${ }^{11}$ The relationship between $\mathrm{EE}$ and the rehospitalization rate of mental illness in Chinese schizophrenia patients has not been addressed.

Recent studies showed that the care of patients can become a significant burden for families, including increased stress, worsened subjective well-being, physical deterioration, and self-value reduction. ${ }^{12}$ Patients' families generally experienced both objective and subjective burden, which can foster family dysfunction. ${ }^{13}$ Lowyck et $\mathrm{al}^{14}$ used the family burden scale to investigate family burden of parents or spouses of schizophrenic patients and found that the family members felt real economic and emotional burden. The severity of burden was closely related to pathological behavior of patients. Liu et $\mathrm{al}^{15}$ found that parents experienced severe burden in taking care of their child with mental illness.

Previous studies have indicated that the EE of the patients' family members is closely related to the subjective burden of disease. ${ }^{16,17}$ However, few studies have discussed the effect of the EE of family members on the care burden of patients with mental illness. This study explored the influence of the EE of caregivers on schizophrenia care burden and rehospitalization rate.

\section{Subjects and methods Study subjects}

A total of 64 schizophrenic patients hospitalized for the first time were recruited from X Hospital from October 2013 to November 2014. In all, 64 key caregivers who lived with the patients for no less than 15 hours each week were recruited. Patients with severe brain and body illnesses, including alcohol or drug dependence, were excluded from study. The sample size was determined using the lookup table method. ${ }^{18}$ According to the study of Möller-Leimkühler and Jandl, ${ }^{17}$ the variation range of correlation coefficient was $0.37-0.69$; 0.4 was designated in this study to evaluate the sample size. Using the lookup table method (1-beta $=0.8$, alpha $=0.05$, bilateral), the estimated sample size was 47 . Considering the loss to follow-up, the initial sample size was set up to 64 . In all, 3 and 11 patients were lost within 6 and 12 months of follow-up, respectively.

\section{Research tools}

The Chinese version of the Camberwell Family Interview (CFI-CV) was used to evaluate the EE of main caregivers. The general data of patients and caregivers were collected. The burden of the caregivers was assessed using the family burden questionnaire.

The EE of caregivers was investigated by the administration of CFI-CV, ${ }^{11}$ which consists of 10 subscales including criticism, hostility, emotion overinvolvement, praise, warmness, resignation, patients' attitude to treatment, family member's caring level, family member's knowledge about disease, and the influence of stigma on patient and family member. Criticism and praise were rated by frequency. The rest of the dimensions were evaluated by scores that varied from 3 to 6 per dimension. HEE is defined when the score of criticism is $\geq 6$ and the score of hostility or emotion overinvolvement is $\geq 3$.

The burden of care in caregivers was assessed using a family burden questionnaire. ${ }^{19}$ The family burden questionnaire includes 14 items, which are divided into three aspects: objective burden, subjective stress burden, and subjective demand burden. Likert five-level score method was used for scoring. A heavy burden of caregivers was defined as objective burden score $>23$, subjective stress burden score $>15$, and subjective demand burden score $>13.5$. The Cronbach's alpha coefficient was 0.86 , and the weight coefficient was 0.87 .

The rehospitalization rate of schizophrenia in patients was defined by the need to be rehospitalized or the increase in dose or type of antipsychotic medications due to symptom recurrence at 6 and 12 months.

Medication Compliance was assessed by self-report of the patients or their key caregivers. Patients or their main caregivers were asked at which time the patients had taken their medications and how many pills the patients had taken for each time. Medication compliance was analyzed according to the following definitions:

- High compliance: following the physicians' order strictly or the days of without following the psychiatrist' order was $<7$ days.

- Low compliance: the period without following the psychiatrist's order was $>1$ week, refusing medication, or discontinuing medication without doctor's permission. The compliance rate was calculated by (the number of patients with high compliance/the number of subjects) $\times 100 \%$. 


\section{Data collection methods}

The data collection was carried out at the first meeting, at 6 and 12 months after discharge by using the same instruments. One research assistant who was a graduate student majored in psychiatric nursing was trained to collect the data. After informed consent, the research assistant conducted face-to-face interview with each main caregiver immediately for the first data collection at the head nurse's office without the patient around. Since CFI-CV was a semi-structured scale, the interview was recorded. After interview, the caregiver was required to fill the family burden questionnaire. Home visits were arranged for local participants to collect follow-up data. For those far away, follow-up data were collected when they came to hospital for rechecks (usually patients were required to recheck every month). The research assistant called the participants to remind their rechecks; if necessary, help was provided to make appointments with the psychiatrists for the patients. For some reasons, if the key caregivers did not come with the patients for rechecks, home visits or phone interviews were conducted to get follow-up data. It took 50-90 minutes for each interview.

\section{Data analysis}

Two persons rated and scored the EE separately according to CFI's guidelines based on the records. When there was inconsistency, the third person was asked to make a judgment. The data were analyzed by using SPSS software. The significance level was set at a $P$-value $<0.05$. Data are expressed as percentage, mean, and standard deviation values. The comparisons of rehospitalization rates and medication compliance between 6 and 12 months were done by Fisher's exact test. Repeated measures analysis of variances (ANOVAs) were used to analyze the change in care burden over time and the influence of EE on care burden and rehospitalization rate.

\section{Ethics}

The protocol for this study has been approved by the ethics committee of Xiangya School of Nursing of Central South University, and the work was undertaken by following the provisions of the Declaration of Helsinki. The signed informed consent forms were obtained from all participants.

\section{Results}

\section{Patterns of caregivers' EE}

The general information of patients and caregivers is listed in Table 1. A total of 46 (71.9\%) caregivers were rated as HEE and $18(28.1 \%)$ caregivers were rated as low expressed
Table I The general information of patients and main caregivers

\begin{tabular}{|c|c|}
\hline Subjects' attributes & n (\%) \\
\hline \multicolumn{2}{|l|}{ Patients } \\
\hline \multicolumn{2}{|l|}{ Gender } \\
\hline Male & $22(34.4)$ \\
\hline Female & $42(65.6)$ \\
\hline \multicolumn{2}{|l|}{ Age, years } \\
\hline$<20$ & $35(54.7)$ \\
\hline $20-50$ & $27(42.2)$ \\
\hline$>50$ & $2(3.1)$ \\
\hline \multicolumn{2}{|l|}{ Marital status } \\
\hline Married & $17(26.6)$ \\
\hline Unmarried & $45(70.3)$ \\
\hline Divorced & I (I.6) \\
\hline Widowed & $\mathrm{I}(\mathrm{I} .6)$ \\
\hline \multicolumn{2}{|l|}{ Vocational status } \\
\hline Student & $35(54.7)$ \\
\hline Worker & $12(18.8)$ \\
\hline Farmer & $2(3.1)$ \\
\hline Leader & $\mathrm{I}(\mathrm{I} .6)$ \\
\hline Jobless & $2(3.1)$ \\
\hline Others & $12(18.8)$ \\
\hline \multicolumn{2}{|l|}{ Educational level } \\
\hline Primary school & $6(9.4)$ \\
\hline Middle school & $21(32.8)$ \\
\hline High school & $17(26.6)$ \\
\hline College degree or above & $20(31.3)$ \\
\hline \multicolumn{2}{|l|}{ Caregivers } \\
\hline \multicolumn{2}{|l|}{ Gender } \\
\hline Male & $20(31.3)$ \\
\hline Female & $44(68.8)$ \\
\hline \multicolumn{2}{|l|}{ Age, years } \\
\hline$<30$ & $4(6.3)$ \\
\hline $30-50$ & $18(76.2)$ \\
\hline$>50$ & II (I7.5) \\
\hline \multicolumn{2}{|l|}{ Marital status } \\
\hline Married & $57(89.1)$ \\
\hline Unmarried & $4(6.3)$ \\
\hline Divorced & $2(3.1)$ \\
\hline Widowed & $\mathrm{I}(\mathrm{I} .6)$ \\
\hline \multicolumn{2}{|l|}{ Vocational status } \\
\hline Worker & $25(39.7)$ \\
\hline Farmer & $14(22.2)$ \\
\hline Leader & $I(I .6)$ \\
\hline Jobless & $3(4.8)$ \\
\hline Others & $20(31.7)$ \\
\hline \multicolumn{2}{|l|}{ Educational level } \\
\hline Primary & $16(28.1)$ \\
\hline Junior middle school & $23(40.4)$ \\
\hline High school & $8(14.0)$ \\
\hline College degree or above & $10(17.5)$ \\
\hline \multicolumn{2}{|l|}{ Residence } \\
\hline Rural & $35(54.7)$ \\
\hline Cities and towns & $29(45.3)$ \\
\hline \multicolumn{2}{|c|}{ Family's monthly income (RMB) } \\
\hline$<3,000$ & $34(54)$ \\
\hline $3,000-7,000$ & $23(36.5)$ \\
\hline$>7,000$ & $6(9.5)$ \\
\hline
\end{tabular}


Table I (Continued)

\begin{tabular}{ll}
\hline Subjects' attributes & $\mathbf{n}(\%)$ \\
\hline Time for patient per day (hours) & \\
$<3$ & $18(28.6)$ \\
$3-6$ & $1 \mathrm{II}(17.5)$ \\
$6-9$ & $\mathrm{II}(17.5)$ \\
$>9$ & $23(36.5)$ \\
Health status of caregiver & \\
Poor & $7(11.1)$ \\
General & $30(47.6)$ \\
Better & $26(41.3)$ \\
Relationship of caregiver and patient & \\
Parents & $47(78.3)$ \\
Spouse & $8(13.3)$ \\
Sibling & $2(3.3)$ \\
Other relatives or non-relatives & $3(5.0)$ \\
\hline
\end{tabular}

Note: RMB is the China Yuan (CNY).

emotion (LEE). Of the 46 HEE caregivers, 18 (34.78\%) were defined as HEE based on their high frequency of criticism comments, $10(21.74 \%)$ were defined as HEE based on the high frequency of emotional overinvolvement, and $20(43.48 \%)$ were defined as HEE due to high frequencies of criticism comments and emotional overinvolvement.

\section{Changes in the burden of care scores over time}

The scores of three dimensions of care burden were decreased significantly over time. Further multiple comparisons showed that the scores at 6 and 12 months post discharge decreased significantly compared to the baseline scores, but no significant differences were found between 6 and 12 months post discharge (Table 2).

\section{Rehospitalization rate and medication compliance}

The rehospitalization rate of patients at 6 months post discharge was $25 \%$, and the medication compliance was $68.2 \%$. The rehospitalization rate of patients at 12 months post discharge was $29.7 \%$, and the rate of medication compliance for 12 months was 59.1\%. The Fisher's exact test showed significant difference in rehospitalization rates and medication compliance between 6 and 12 months $(P=0.06$ and $P=0.000$, respectively; Table 2 ). A significant difference was found by the Fisher's exact test in the rehospitalization rates between patients with high medication compliance and low medication compliance at 12 months $(P=0.003)$ but not at 6 months post discharge $(P=0.056)$.

\section{Influence of different EE levels on care burden and rehospitalization rate of schizophrenia}

As shown in Table 3, the time statistically significantly influenced the objective burden, subjective stress burden, and subjective demand burden but not the rehospitalization rate. The EE levels statistically significantly influenced the subjective stress burden, indicating difference in subjective stress burden between HEE and LEE, and the rehospitalization rate without interaction with time. There was a statistically significant interaction between EE levels and time, which affected only objective burden (Table 3).

Significant high rehospitalization rate was observed in patients supported by caregivers with HEE compared to those assisted by caregivers with LEE without any statistically significant interaction between $\mathrm{EE}$ and time. Further analysis of different components of EE showed that the rehospitalization rate was statistically different between the high and low levels of criticism comments ( $F=0.049, P=0.033)$. No statistical difference in the rehospitalization rates was observed between high emotional overinvolvement and low emotional overinvolvement ( $F=0.480, P=0.493$; Figure 1).

\section{Discussion}

This study highlighted that $71.9 \%$ of caregivers of patients affected by schizophrenia presented HEE mainly represented by criticism $(34.78 \%)$. This result shows a higher prevalence of HHE in comparison with previous studies that reported that the percentage ranged from $48.6 \%$ to $54 \%$ of schizophrenia patients who lived with HEE relatives. ${ }^{20,21}$ The high frequency of criticism, observed in our study, is consistent with other research,

Table 2 Results of care burden, rehospitalization rate, and medication compliance at different time points

\begin{tabular}{llllll}
\hline Variables & Baseline & Sixth month & Twelfth month & Statistic value & $P$-value \\
\hline Subjective demand burden & $14.162 \pm 0.402$ & $12.703 \pm 0.222$ & $12.351 \pm 0.170$ & $9.357^{\mathrm{a}}$ & 0.000 \\
Subjective stress burden & $15.973 \pm 0.362$ & $14.000 \pm 0.363$ & $14.081 \pm 0.304$ & $14.246^{\mathrm{a}}$ & 0.000 \\
Objective burden & $20.622 \pm 0.489$ & $18.676 \pm 0.386$ & $18.459 \pm 0.383$ & $14.578^{\mathrm{a}}$ & 0.000 \\
Rehospitalization rate (\%) & & 25 & 29.7 & $0.006^{\mathrm{b}}$ \\
Medication compliance (\%) & & 68.2 & 59.1 & $0.000^{\mathrm{b}}$ \\
\hline
\end{tabular}

Notes: ${ }^{a} F$ value from repeated measures ANOVAs. 'P $P$-value from Fisher's exact test. Abbreviation: ANOVA, analysis of variance. 
Table 3 The influence of different patterns of EE on care burden and rehospitalization rate

\begin{tabular}{|c|c|c|c|c|c|c|}
\hline \multirow[t]{2}{*}{$\begin{array}{l}\text { Care burden/ } \\
\text { rehospitalization rate }\end{array}$} & \multicolumn{2}{|c|}{ Main effect of time } & \multicolumn{2}{|c|}{ Main effect of EE } & \multicolumn{2}{|c|}{$\begin{array}{l}\text { Interaction between } \\
\text { time and } \mathrm{EE}\end{array}$} \\
\hline & $F^{*}$ & $P$-value & $F^{*}$ & $P$-value & $F^{*}$ & $P$-value \\
\hline Objective burden & 13.080 & 0.000 & 0.76 & 0.389 & 3.604 & 0.032 \\
\hline Subjective stress burden & 10.652 & 0.000 & 8.012 & 0.008 & 0.485 & 0.618 \\
\hline Subjective demand burden & $11.67 \mid$ & 0.000 & 0.929 & 0.342 & 0.635 & 0.533 \\
\hline Rehospitalization rate & 0.095 & 0.760 & 7.336 & 0.010 & 0.575 & 0.453 \\
\hline
\end{tabular}

Notes: *Repeated measures ANOVAs. The significant influence existed when $P<0.05$ (shown in bold).

Abbreviations: EE, expressed emotion; ANOVA, analysis of variance.

which highlighted that, in China, criticism contributes the most to EE. ${ }^{22}$ This may be associated with the following hypothesizes: 1) most of our patients were at first-episode schizophrenia and had been hospitalized due to severe symptoms; 2) main caregivers did not know the mental illness, particularly, schizophrenia; and 3 ) the small sample size may have caused some bias.

\section{A}

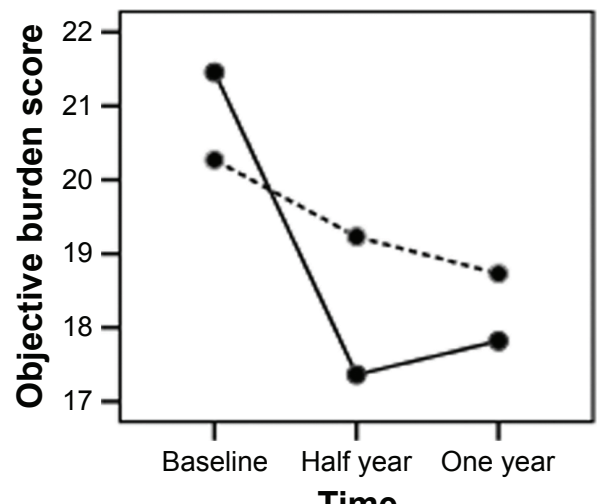

Time
Consistent with previous studies, ${ }^{14,15}$ we highlighted that the subjective stress burden and subjective demand burden scores were significantly higher in caregivers either when the schizophrenia patients were hospitalized or at 6 and 12 months after discharge compared to the baseline. Moreover, there were differences in the scores of objective

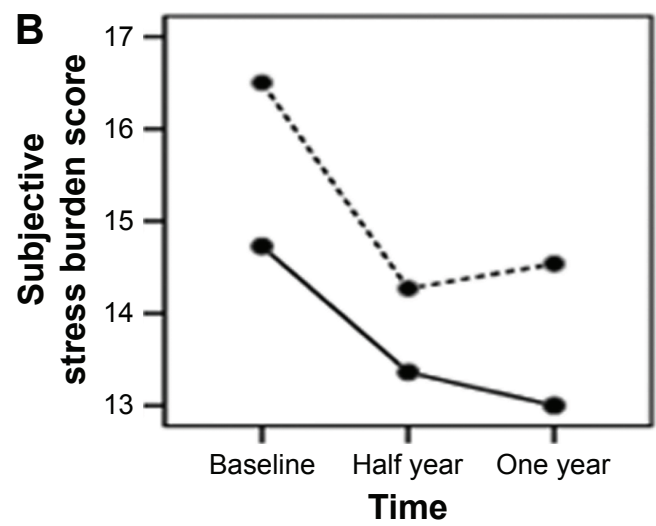

Level of expressed emotion of caregivers - LEE .... HEE
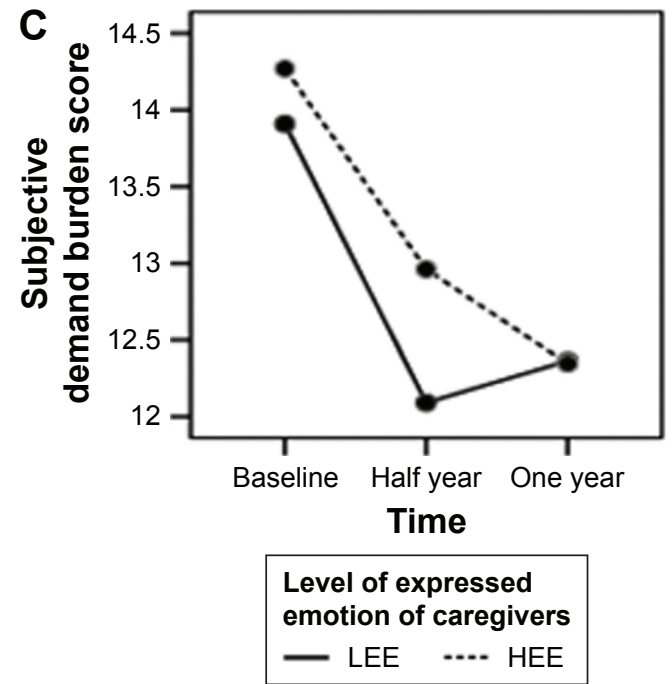
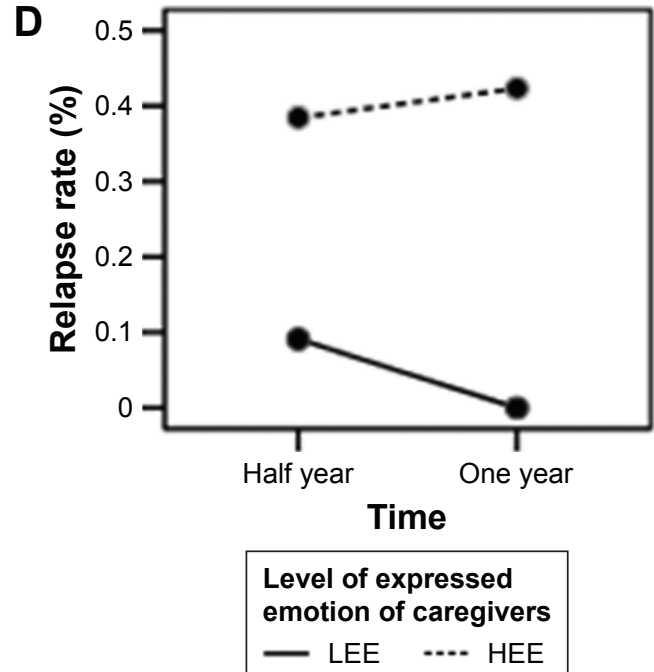

Figure I The correlation analysis.

Notes: (A) The association between objective burden and time. (B) The association between subjective stress burden and time. (C) The association between subjective demand burden and time. (D) The association between rehospitalization rate and time.

Abbreviations: LEE, low expressed emotion; HEE, high expressed emotion. 
burden, subjective demand burden, and subjective stress burden between different time points. Although the burden of care scores at 6 and 12 months after discharge was reduced compared to those reported at hospitalization, it was higher than that at baseline. This might be associated with the following hypotheses: 1) when patients were hospitalized, their primary caregivers were under great economic and emotional stress; with the improvement in the patient's condition, the stress on the family was gradually reduced and the burden of caregivers was correspondingly reduced; 2) some family members of patients were troubled by the stigma of mental illness, being afraid of telling others and worrying about being prejudiced and discriminated by other people; and 3) our patients were hospitalized for the first time and most of them did not return to the society in a short time; therefore, they needed care from their family.

Many studies have demonstrated that medication adherence is an important factor affecting the rehospitalization of schizophrenia patients. However, the condition of medication adherence of schizophrenia patients is not optimistic. In contrast, medication adherence may be affected by longterm medication, economic stress, patient's insight, cognitive function, and other factors. For example, Mi et $\mathrm{al}^{23}$ reported that rehospitalization rates of schizophrenia patients with high medication adherence and low medication adherence were statistically different, suggesting a correlation between the medication adherence and rehospitalization rate. In this study, the rehospitalization rates were $25 \%$ and $29.7 \%$ at 6 and 12 months and the adherence rates were $68.2 \%$ and $59.1 \%$ for patients at 6 and 12 months post discharge, respectively. A significant difference was found in the rehospitalization rates between patients with high medication compliance and low medication compliance at 12 months but not at 6 months post discharge. The result proved that the rehospitalization rate was correlated with medication compliance; the longer the medication time, the poorer the medication compliance and the higher the rehospitalization rate.

Consistent with previous studies, ${ }^{24,25}$ which suggested that EE was a reliable predictor of schizophrenia rehospitalization, this study showed that the rehospitalization rate of patients with HEE caregivers was higher than those with LEE caregivers. In addition, the higher level of criticism was related to higher rehospitalization rate. In addition, EE's effect on rehospitalization is independent of the effects of the social demographic characteristics of caregivers, disease characteristics of patients, treatment compliance, and other factors' effects on rehospitalization. ${ }^{26}$ This study showed that there was no interaction between time and emotion, so that the $\mathrm{EE}$ in predicting outcome was independent of the time factor. The different components of EE, such as criticism and emotional overinvolvement, play different roles in predicting the rehospitalization rate. In this study, levels of criticism had a large effect on the rehospitalization rate.

The subjective burden is the psychological feeling of burden in family members of the patients. The objective burden is the burden caused by the patients' disease becoming a disturbing factor in family life and is caused by adverse events. ${ }^{27}$ Breitborde et $\mathrm{al}^{24}$ suggested that the EE and the burden of caregivers were closely related. Möller Leimkühler ${ }^{28}$ concluded that EE was one of the most relevant predictors of families' burden. Giuseppe et $\mathrm{al}^{29}$ showed that the family members with HEE reported more subjective burden related to patients' behavioral disorders. This study showed that the subjective stress burden score was greater in HEE caregivers than in LEE caregivers, while there was no significant difference in the objective burden and subjective demand burden scores between HEE and LEE caregivers.

There are several limitations to this study. First, this study only included patients with severe mental illness who had required hospitalization. Second, the majority of primary caregivers were women (70\%) and were the patients' mothers. A previous study suggested that men and women experienced different care burden, but women experienced a higher level of burden and a greater risk of mental problems. ${ }^{30}$ It is unclear whether the conclusion will be changed if the caregivers are a group of professional caregivers, male caregivers, or younger caregivers. Third, although the sample size of the study met the required sample size for the study, it is small and can cause bias.

\section{Conclusion}

This study highlighted that the EE of primary caregivers was a predictor of rehospitalization rate in schizophrenia patients and the primary caregivers of schizophrenia patients had an obvious burden of care. This study showed concerns about the caregivers, especially for caregivers with HEE. The intervention should be strengthened, such as educational interventions tackling mental illness and psychotherapy support to help caregivers to be more sympathetic and tolerant toward patients.

\section{Implications for practice}

The results of this study implicate that continuing care outside hospital is necessary to facilitate the recovery of patients with schizophrenia because the rehospitalization rate increased and the medication compliance decreased over time. Findings also highlight the need of family interventions that provide proper information and psychological support to help relatives improve their understanding of the disorder, handle 
difficult thoughts and emotions, reduce negative appraisals, and prevent HEE over the psychotic process. In this study, caregiver's EE was investigated only by their own description and behaviors, but if the patients perceived the caregiver's EE in the same way, further study will be needed in the future.

\section{Acknowledgments}

The authors acknowledge the financial support by the Chinese Medical Board (10-020-201201). Thanks to Prof Ke Hengming for proofreading (Prof Ke works at the School of Medicine, University of North Carolina, USA; email address: Hengming Ke@med.unc.edu).

\section{Disclosure}

The authors report no conflicts of interest in this work.

\section{References}

1. Di Lorenzo R, Sagona M, Landi G, Martire L, Piemonte C, Del Giovane C. The revolving door phenomenon in an Italian acute psychiatric ward: a 5-year retrospective analysis of the potential risk factors. J Nerv Ment Dis. 2016;204(9):686-692.

2. Brown GW, Birley JL, Wing JK. Influence of family life on the course of schizophrenic disorders: a replication. Br J Psychiatry. 1972; 121(562):241.

3. Brown GW, Rutter M. The measurement of family activities and relationships: a methodological study. Human Relat. 1966;2(suppl 3): 241-263.

4. Li Z. Research progress in emotional expression of family members of patients with schizophrenia. Chin J Nurs. 2002;37(12):927-929.

5. Leff JP, Vaughn C. Expressed Emotion in Families: Its Significance for Mental Illness. New York: Guilford Press; 1985.

6. Cutting LP, Aakre JM, Docherty NM. Schizophrenic patients' perceptions of stress, expressed emotion, and sensitivity to criticism. Schizophr Bull. 2006;32(4):743.

7. Hooley JM, Gotlib IH. A diathesis-stress conceptualization of expressed emotion and clinical outcome. Appl Prev Psychol. 2000;9(3):135-151.

8. Linszen DH, Dingemans PM, Nugter MA, Van der Does AJ, Scholte WF, Lenior MA. Patient attributes and expressed emotion as risk factors for psychotic relapse. Schizophr Bull. 1997;23(1):119-130.

9. Bhugra D, Mckenzie K. Expressed emotion across cultures. Adv Psychiatr Treat. 2003;18(9):342-348.

10. Hashemi AH, Cochrane R. Expressed emotion and schizophrenia: a review of studies across cultures. Int Rev Psychiatry. 2009;11(2-3): 219-224.

11. Phillips MR, Xiong W. Expressed emotion in mainland China: Chinese families with schizophrenic patients. Int $J$ Ment Health. 1995;24(3):54-75.

12. Hirst M. Carer distress: a prospective, population-based study. Soc Sci Med. 2005;61(3):697-708.
13. Li J, Xiang MZ, Guo LT, et al. Comparative study on family burden of patients with schizophrenia in urban and rural areas of China. West Chin Med. 1998;13(1):426.

14. Lowyck B, De Hert M, Peeters E, Wampers M, Gilis P, Peuskens J. A study of the family burden of 150 family members of schizophrenic patients. Eur Psychiatry. 2004;19(7):395-401.

15. Liu M, Lambert CE, Lambert VA. Caregiver burden and coping patterns of Chinese parents of a child with a mental illness. Int J Ment Health Nurs. 2007;16(2):86-95.

16. Möller-Leimkühler AM, Obermeier M. Predicting caregiver burden in first admission psychiatric patients. Eur Arch Psychiatry Clin Neurosci. 2008;258(7):406.

17. Möller-Leimkühler AM, Jandl M. Expressed and perceived emotion over time: does the patients' view matter for the caregivers' burden? Eur Arch Psychiatry Clin Neurosci. 2011;261(5):349-355.

18. Nancy BPD, Grove SK. The Practice of Nursing Research: Conduct, Critique, \& Utilization (4th edition). New York, W.B. Saunders Company, 2001:89.

19. Montgomery RJ. Using and interpreting the Montgomery Borgatta Caregiver Burden Scale. 2002. Available from: http://www4.uwm.edu/ hbssw/PDF/Burden\%20Scale.pdf. Accessed August 15, 2017.

20. Chen ZJ, Fan H, Wang Y, et al. The relationship between emotional expression and personality traits in the first degree relatives of schizophrenic patients. Clin Psychiatr Med. 2012;22(4):252-254.

21. Bebbington $P$, Kuipers L. The clinical utility of expressed emotion in schizophrenia. Acta Psychiatr Scand Suppl. 1994;382:46-53.

22. Cechnicki A, Bielańska A, Hanuszkiewicz I, Daren A. The predictive validity of expressed emotions (EE) in schizophrenia. A 20 -year prospective study. J Psychiatr Res. 2013;47(2):208.

23. Mi WF, Zou LY, Li ZM, et al. Compliance with antipsychotic treatment and relapse in schizophrenia. Chin J Psychiatry. 2012;45(1):25-28.

24. Breitborde NJ, López SR, Wickens TD, Jenkins JH, Karno M. Toward specifying the nature of the relationship between expressed emotion and schizophrenic relapse: the utility of curvilinear models. Int $J$ Methods Psychiatr Res. 2007;16(1):1-10.

25. Aguilera A, López SR, Breitborde NJK, Kopelowicz A, Zarate R. Expressed emotion and sociocultural moderation in the course of schizophrenia. J Abnorm Psychol. 2010;119(4):875-885.

26. Heikkilä J, Karlsson H, Taiminen T, et al. Expressed emotion is not associated with disorder severity in first-episode mental disorder. Psychiatry Res. 2002;111(2):155-165.

27. Lu F, Zeng G. Theoretical framework and its development of the evaluation of disease burden. Chin J Epidemiol. 2001;22(4):259-261

28. Möller Leimkühler AM. Burden of relatives and predictors of burden. Baseline results from the Munich 5-year-follow-up study on relatives of first hospitalized patients with schizophrenia or depression. Eur Arch Psychiatry Clin Neurosci. 2005;255(4):223-231.

29. Giuseppe C, Lorenzo CC, Massimo C. The association between expressed emotion, illness severity and subjective burden of care in relatives of patients with schizophrenia: findings from an Italian population. BMC Psychiatry. 2012;12(1):1-8.

30. Yee JL, Schulz R. Gender differences in psychiatric morbidity among family caregivers: a review and analysis. Gerontologist. 2000;40(2): $147-164$.
Patient Preference and Adherence

\section{Publish your work in this journal}

Patient Preference and Adherence is an international, peer-reviewed, open access journal that focuses on the growing importance of patient preference and adherence throughout the therapeutic continuum. Patient satisfaction, acceptability, quality of life, compliance, persistence and their role in developing new therapeutic modalities and compounds to optimize

\section{Dovepress}

clinical outcomes for existing disease states are major areas of interest for the journal. This journal has been accepted for indexing on PubMed Central. The manuscript management system is completely online and includes a very quick and fair peer-review system, which is all easy to use. Visit http://www. dovepress.com/testimonials.php to read real quotes from published authors. 